\title{
Mid-IR Enhanced Laser Ablation Molecular Isotopic Spectrometry
}

\author{
Staci Brown ${ }^{1, \dagger}$, Alan Ford ${ }^{2,{ }^{\star}}$ Codjo A. Akpovo, ${ }^{1}$ and Lewis Johnson ${ }^{1, \dagger}$ \\ ${ }^{1}$ Department of Physics, Florida A\&M University, 2077 E. Paul Dirac Drive, Tallahassee, FL
} 32310

${ }^{2}$ Alakai Defense Systems, 197 Replacement Ave, Suite 102, Fort Leonard Wood, MO 65473

*Corresponding author: alan.ford@alakaidefense.com, ph. 573-329-1919

†Additional Author emails: Staci Brown, staci.brown@cepast.famu.edu, ph. 850-599-8470;

Codjo A. Akpovo, c.akpovo@famu.edu, ph. 850-599-8470; Lewis Johnson, lewis@cepast.famu.edu, ph. 850-599-8456

\begin{abstract}
A double-pulsed laser-induced breakdown spectroscopy (DP-LIBS) technique utilizing wavelengths in the mid-infrared (MIR) for the second pulse, referred to as double-pulse LAMIS (DP-LAMIS), was examined for its effect on detection limits compared to single-pulse laser ablation molecular isotopic spectrometry (LAMIS). A MIR carbon dioxide $\left(\mathrm{CO}_{2}\right)$ laser pulse at $10.6 \mu \mathrm{m}$ was employed to enhance spectral emissions from nanosecond- laser-induced plasma via mid-IR reheating and in turn, improve the determination of the relative abundance of isotopes in a sample. This technique was demonstrated on a collection of ${ }^{10} \mathrm{BO}$ and ${ }^{11} \mathrm{BO}$ molecular spectra created from enriched boric acid $\left(\mathrm{H}_{3} \mathrm{BO}_{3}\right)$ isotopologues in varying concentrations. Effects on the overall ability of both LAMIS and DP-LAMIS to detect the relative abundance of boron isotopes in a starting sample were considered. Least-squares fitting to theoretical models was used to deduce plasma parameters and understand reproducibility of results. Furthermore, some optimization for conditions of the enhanced emission was achieved, along with a comparison of the overall emission intensity, plasma density, and plasma temperature generated by the two techniques.
\end{abstract}

\section{Introduction}

Recently, it has been proposed that isotopic information from a sample can be obtained via laser-induced breakdown spectroscopy (LIBS) by what is known as laser-ablation molecular isotopic spectrometry (LAMIS) [1-3]. In the LAMIS method, the rovibronic emission spectra of 
diatomic molecules produced in the LIBS plasma are shifted by the effect of the different isotopic masses on rovibronic energies [4,5]. Using these shifts, the relative abundance of isotopes in a normally solid sample can be determined $[6,7]$.

While the original LAMIS research involved single-pulse LIBS, multi-pulse LIBS techniques for LAMIS have been suggested to lower limits of detection for a given element [2], and they could possibly offer more reliable determination of the isotopes of interest. The most common multipulse technique, which consists of two pulses separated in time, is termed double-pulse LIBS (DP-LIBS), as the second pulse is generally believed to be another breakdown and reheating of the first event [8-15]. Previous studies have shown that DP-LIBS techniques increase the overall emission from a plasma by about an order of magnitude, and thereby leading to better sensitivity for the detection of a given element [10,16-18]. Moreover, multi-pulse techniques have been shown to reduce the pulse-to-pulse variation inherent to single pulse LIBS, which improves the repeatability of LIBS experiments $[19,20]$.

Multi-pulse LIBS can involve a variety of beam orientations and timings with their own designation, such as pre-ablative-spark LIBS [21,22], collinear DP-LIBS [23], and orthogonal DP-LIBS [24-26]. Any one of these techniques can potentially be used to enhance the LAMIS emission with the possibility of improving the ability to detect the relative abundance of isotopes (more specifically, those of lower abundance) by increasing their emission relative to the inherent noise in LIBS spectra. This concept was demonstrated by Mao et al. [2] with orthogonal DP-LIBS using two similar $355 \mathrm{~nm}$ lasers for LAMIS. Even so, the conditions that optimize the emission for a given species are not always apparent, principally because the exact experimental parameters, such as laser wavelengths, pulse energies, delay between pulses, offset of breakdown from the target surface, and other settings, are not absolute for these techniques [27-29]. The net result is that LAMIS acquires the same complexity as the LIBS method used to create the plasma, albeit the complexity allows for a great deal of customization that can improve the detection performance under controlled conditions.

One of these customizations is the signal-enhancing DP-LIBS technique denoted Townsend effect plasma spectroscopy (TEPS) [30]. TEPS is a double-pulse technique in which the second laser (the reheating pulse) has much longer wavelength and wider pulse length than the one producing the plasma. The TEPS method does not try to recreate a breakdown on the ablated material from the first LIBS pulse or cause a second LIBS event on the same surface as the first pulse of LIBS. Instead, a non-ablative, defocused or collimated, second laser pulse, in the midinfrared $(\mathrm{IR})$ region - typically a carbon dioxide $\left(\mathrm{CO}_{2}\right)$ transversely excited atmospheric (TEA) laser operating at $10.6 \mu \mathrm{m}$ - is used to re-excite the plasma [30-32]. Whereas DP-LIBS routinely provides enhancements close to an order of magnitude, TEPS can create enhancements close to two orders of magnitude in some cases under long irradiance [33,34]. Though not certain, the larger enhancement is possibly due to plasma reheating in an airrarefied region, as is common in DP-LIBS using only near-IR wavelengths [32,35-37]. It should be noted that TEPS likely does not rely on the electron scattering of the Townsend effect, as suggested by the acronym; however, the exact nature of the reheating process is not clear though it has been studied in the past $[32,38]$. Regardless, the long interaction time of the TEA laser that is associated with TEPS (commonly, 50\% energy in a 100 to 200 ns peak with 
another $50 \%$ in a 1 to $10 \mu$ s tail), as well as the sustainment of the plasma for a longer period than for DP-LIBS with only near-IR wavelengths makes TEPS an alternative and potentially superior technique for creating ionic, atomic, and molecular species from a starting LIBS plasma $[34,39]$. Consequently, TEPS could provide even greater sensitivity over other DP-LIBS methods, making it useful for understanding its application to LAMIS. Nevertheless, the precise effect of double-pulse techniques such as TEPS on the emission from diatomics as desired for LAMIS is not widely available and is somewhat unclear. As an example, nanosecondnanosecond (ns-ns) near-IR DP-LIBS of boron nitride shows increased emission from boron monoxide $(\mathrm{BO})$ in a follow up paper to the original LAMIS work [2]. Others have seen similar enhancements for ns-ns DP-LIBS using two IR lasers applied to the detection of explosives and halide molecules [40,41]. On the contrary, a DP-LIBS investigation using femtosecond (fs) LIBS as the ablative pulse followed by a second fs pulse produced increased emission from neutrals and ions but not molecules [42]. These results suggest that the emission from molecules may depend on longer plasma-laser interaction, which would be advantageous for TEPS. In addition, the mid-IR wavelength of the second pulse of TEPS has the potential for more coronal and atmospheric plasma heating possibly leading to subsequent conduction of heat into the plasma $[43,44]$; though when the plasma density is high, it couples into the plasma less efficiently than the near-IR wavelengths of DP-LIBS more commonly used [44]. This conductive heating would maintain the plasma over an extended period, would further extend the plasma lifetime, and if molecular formation relates to plasma lifetime, would contribute to more molecule formation.

More specific to molecular emission is a recent study of polystyrene and TNT films where a $\mathrm{CO}_{2}$ TEA laser was used as the second pulse in DP-LIBS after $\mathrm{Nd}^{3+}: Y A G-l a s e r$ LIBS [39]. The polystyrene films revealed an increase in emission of $C N$ and $\mathrm{C}_{2}$ under atmospheric conditions. However, the experiment on films of trinitrotoluene (TNT) showed no increase in $\mathrm{C}_{2}$ emission, but trended to more intense $\mathrm{CN}$ features. These results suggest that chemical reactions with atmosphere initiated by the addition of the long IR pulse of DP-LIBS can create enhanced molecular emission [43,44], but enhancement may depend on the initial mechanisms of breakdown [39]. For the two cases here, a given IR power density likely facilitated the evaporation and explosive decomposition or burning of TNT, which has been personally observed by the current authors and others studying this effect [45-47], but polystyrene simply fragmented due to better chemical stability, as evidenced by the melting and boiling points of these two materials. Trinitrotoluene has an $80^{\circ} \mathrm{C}$ melting point and a $240^{\circ} \mathrm{C}$ explosive boiling point; polystyrene has a melting point greater than $100^{\circ} \mathrm{C}$ m.p. but starts to decompose around $200^{\circ} \mathrm{C}$ or greater. Though other factors such as reflectivity and thermal absorption are important [45-47], it is possible the limited amount of supplied energy enabled more energy transfer to the bulk TNT than bulk polystyrene, leading to more energized ablation products. It is the products of the initial reactions that likely reacted with the excited atmosphere to give some of the molecules that were observed. However, the conductive heating of the mid-IR laser should maintain the plasma over an extended period, as well as further extend the plasma lifetime. If molecular formation relates to plasma lifetime, the heating should additionally contribute to more molecule formation, including more molecular the oxides that are primarily used by LAMIS. However, a similar experiment on films of trinitrotoluene (TNT) showed no increase in $\mathrm{C}_{2}$ emission, but trended to more intense $\mathrm{CN}$ features. These results suggest that chemical 
reactions with atmosphere initiated by the addition of the long IR pulse of TEPS can create enhanced molecular emission, but enhancement may depend on the initial mechanisms of breakdown [39]. For the two cases here, a given IR power density likely facilitated the explosive decomposition or burning of TNT, but polystyrene simply fragmented due to better chemical stability. It is the products of these initial reactions that reacted with the excited atmosphere to give some of the molecules that were observed.

The formation of larger amounts of molecules by atmospheric reaction in some cases would seem to make TEPS ideal for improving LAMIS, but this idea is not necessarily true. The increased number density of emitting molecules should improve the signal-to-noise over LIBS and other DP-LIBS techniques, leading to lower detection limits for an element in general and improving the ability to quantify lower percentages of an isotope in a sample relative to those in higher abundance. Regardless, at least one study illustrates that when plasmas from SP-LIBS and DP-LIBS with lower ablation energy have about the same intensities but only slightly different plasma parameters (i.e., temperature, electron density, etc.) [48], there is little advantage from multi-pulse techniques in quantitative LIBS analyses for elements. This latter result implies that if a TEPS-created plasma does not have very different characteristics than its LIBS counterpart, quantitative analyses of elements will benefit little from TEPS, but it is hard to say if the same can be said of molecules or, in particular, isotopologues, thus meriting further study for this technique for LAMIS. To investigate possible TEPS enhancements on LAMIS, isotopic analysis of boric acid $\left(\mathrm{H}_{3} \mathrm{BO}_{3}\right)$ isotopologues via LAMIS was performed along with a LAMIS analysis using a $\mathrm{CO}_{2}$ TEA laser for enhancement of LIBS to produce TEPS. The latter technique is hereafter referred to simply as DP-LAMIS without regards to the wavelength of the enhancement laser. Effects on the overall ability of the methods to detect the relative abundance of boron isotopes in the starting sample were noted. Furthermore, some optimization for conditions of the TEPS emission was achieved, along with a comparison of the overall emission intensity, plasma density, and plasma temperature generated by the two techniques.

\section{Methods and Materials}

A simplified diagram of the instrument used for LAMIS analysis is shown in Figure 1. A Qswitched, $\mathrm{Nd}^{3+}$ :YAG laser (Quantel Brilliant B) operating at $532 \mathrm{~nm}$ with $100 \mathrm{~mJ}$ of output energy and $\sim 5$ ns pulse duration generated the LIBS plasma. The beam was expanded, collimated, and focused behind the target using an achromatic lens (f.l. $250 \mathrm{~mm}$ ) for a spot size of $1 \mathrm{~mm}$ at the sample surface after passing through a periscopic assembly. A photodiode, detecting the plasma at onset, was linked to an oscilloscope to monitor the laser-induced light and well as detector settings (e.g., gate width and gate delay) for better synchronization of plasma event and acquisition. A helium-neon laser line level was used to ensure that all samples maintain the same lens to sample distance. An Andor iCCD camera (DH740-25F-03, Andor Technology) attached to an Acton spectrograph (SpectraPro 750i, $3600 \mathrm{gr} / \mathrm{mm}, 240 \mathrm{~nm}$ blazed) was used to acquire and visualize the spectral emissions. In the study, the BO B-system, $\mathrm{B}^{2} \Sigma^{+} \rightarrow \mathrm{X}^{2} \Sigma^{+}$in $255-$ $265 \mathrm{~nm}$ wavelength ranges was investigated. The operating gate delay and width were respectively $25 \mu \mathrm{s}$ and $35 \mu \mathrm{s}$. 


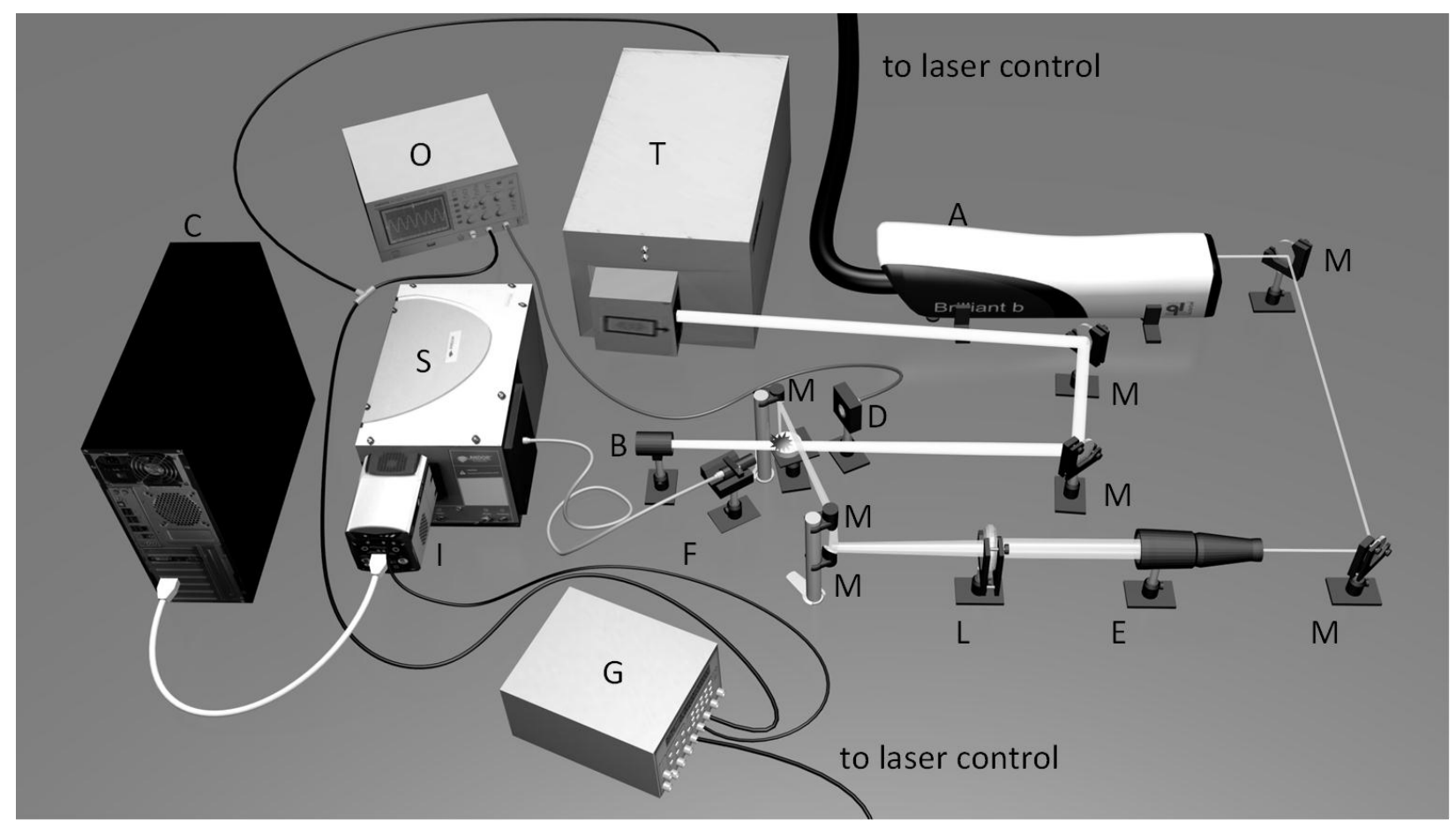

Figure 1. Experimental setup for the LAMIS and DP-LAMIS analysis used in this work. In the figure, the symbols are as follows: $\mathrm{A}=$ laser, $\mathrm{B}=$ beam dump, $\mathrm{C}=$ computer, $\mathrm{D}=$ photodiode, $\mathrm{E}=$ beam expander, $\mathrm{F}=$ fiber optic collimating lens, $\mathrm{G}=$ digital-delay generator, I=iCCD camera, $\mathrm{L}=$ lens, $\mathrm{M}=$ mirror, $\mathrm{O}=$ oscilloscope, $\mathrm{S}=$ spectrometer, and $\mathrm{T}=\mathrm{CO}_{2} \mathrm{TEA}$ laser. The digital-delay generator also times the laser through a laser-control box, which is not shown. This box is connected to the laser by an umbilical, part of which is detailed in the figure.

Crossing the ablation pulse, and $0.5 \mu$ s after this later, was a $\mathrm{CO}_{2}$ TEA laser beam from a highpower, Q-switched $\mathrm{CO}_{2}$ TEA laser (Lumonics Model 920; 3.5J/pulse) operating at $10.6 \mu \mathrm{m}$ and $10 \mathrm{~Hz}$. The square $\mathrm{CO}_{2}$ TEA laser beam was rotated $45^{\circ}$ by periscopic manipulation before being routed parallel to the sample surface across the plasma. The beam was focused with a zinc selenide ( $\mathrm{ZnSe}$ ) lens of $30 \mathrm{~cm}$ focal length giving a $\sim 25 \mathrm{~mm}^{2}$ cross-section at the plasma interaction point, which corresponded to a power density of $0.32 \mathrm{MW} \cdot \mathrm{cm}^{-2}$. The parallel configuration prevented additional ablation of the target compared to LIBS. Because of the longer plasma decay that occurs with the $\mathrm{CO}_{2}$ TEA interaction, two gate delays of the iCCD were used for DP-LAMIS: $25 \mu \mathrm{s}$, the same delay as for LAMIS, denoted DP-LIBS ${ }_{25}$ or DPLAMIS $_{25}$ and $35 \mu$ s denoted as DP-LIBS ${ }_{35}$ or DP-LAMIS ${ }_{35}$.

The analytes used throughout were isotopologues of $\mathrm{H}_{3} \mathrm{BO}_{3}$ ground in a mortar to make about $0.5 \mathrm{~g}$ of total material to produce specimens of a certain molar percentage of a given isotopologue. Isotopically-pure $\mathrm{H}_{3} \mathrm{BO}_{3}$ or natural abundance $\mathrm{H}_{3} \mathrm{BO}_{3}$ from Cambridge Isotopes or Sigma-Aldrich (listed in Table 1) were measured using an analytical balance accurate to $0.1 \mathrm{mg}$ before adding to a mortar. The material was then ground by hand with a pestle for about 10 minutes to produce a fine powder. Samples of $2.5,5.0,10.0,25.0,50.0,75.0,90.0,95.0$, and $97.5 \%{ }^{10} \mathrm{~B}$, along with the pure isotopologue samples were prepared. 
Table 1: Materials used in this research

\begin{tabular}{lllll}
\hline \multicolumn{1}{c}{ Chemical } & \multicolumn{1}{c}{ Supplier } & \multicolumn{1}{c}{ Part Number } & \multicolumn{1}{c}{ Purity } & \multicolumn{1}{c}{ Isotopic Purity } \\
\hline $\mathrm{H}_{3}{ }^{10} \mathrm{BO}_{3}$ & Cambridge Isotopes & BLM-870-0 & $98 \%$ & $99 \%{ }^{10} \mathrm{~B}$ \\
$\mathrm{H}_{3}{ }^{11} \mathrm{BO}_{3}$ & Cambridge Isotopes & BLM-797-0 & $98 \%$ & $99 \%{ }^{11} \mathrm{~B}$ \\
$\mathrm{H}_{3} \mathrm{BO}_{3}$ & Sigma-Aldrich & 202878 & $100 \%$ & nat. abund. \\
$\mathrm{D}_{3} \mathrm{BO}_{3}$ & Sigma-Aldrich & 343846 & $98 \%$ & $98 \% \mathrm{D}$ \\
\hline
\end{tabular}

Pellets of the pure or mixed samples were produced by compressing the powders under approximately $160 \mathrm{MPa}$ for $30 \mathrm{~min}$ in a hydraulic pellet compressor (Carver, Inc., USA) to an average diameter of $13 \mathrm{~mm}$ and an average thickness of 2.41 to $4.71 \mathrm{~mm}$. After removal from the press, LIBS or TEPS spectra were taken at 50 different locations on each pellet, with each location being sequentially sampled 30 times at identical instrument settings and summed together. Data collection was accomplished in approximately one day for a given series of concentrations of ${ }^{10} \mathrm{~B}$ (about 30 min per pellet), which minimized instrument drift for that series. However, data were taken on different days separated by up to three months, so that instrumental and environmental variation could be determined; this separation in time was considered vital for creating reliable models that could be used over several months without recalibration.

The initial analysis of each material was carried out by least squares fitting to simulate rovibronic emission spectra of the $\mathrm{B} \rightarrow \mathrm{X}$ transitions of $\mathrm{BO}$ isotopologues (i.e., ${ }^{10} \mathrm{~B}^{16} \mathrm{O}$ or ${ }^{11} \mathrm{~B}^{16} \mathrm{O}$ ). Transition energies were calculated using algebraic expressions for the reduced Hamiltonian [1,50-57] and constants from Mélen et al. [58]. Intensities of each transition were calculated according to the equations found in da Silva [55], with transition strengths and Franck-Condon factors coming from Ochkin [59] and Liszt et al. [60] respectively. Each transition was convoluted with a mixture of Gaussian and Lorentizan profiles that approximated a Voigt line shape, which encompassed the pressure, Stark, Doppler, and spectrometer-broadening mechanisms [61-65]. The FWHM for this line shape was determined to be about $4 \mathrm{~cm}^{-1}$ using Monte-Carlo simulations. Least squares fitting routines provided by Matlab software [66] accomplished the fit to experimental spectra by changing the intensities of the theoretical spectra of the two isotopologues, the local thermodynamic equilibrium (LTE) temperature of the plasma, and the offset of the spectrum created by adding the individual contributions of the isotopologues. Further details describing this initial analysis method can be found in a previous work.[67]

Because of demonstrated improvements seen in the accuracy for relative abundance analysis when utilizing more heuristic approaches to LAMIS, partial least squares (PLS) and classical least squares (CLS) models were also constructed for $\%{ }^{10} \mathrm{~B}$ determination for each pellet. Both these techniques relied on the tools available in the PLS_Toolbox from Eigenvector [68]. Supervised training of each model was accomplished by using the first 10 LIBS or TEPS spectra from each pellet at a given gate delay as calibration data and the other shots from the respective technique at the same gate delay as validation data; these data formed the X-block. The optimal number of loading vectors for PLS was chosen by using a contiguous-block crossvalidation; three loading vectors were used because this combination gives the minimum root- 
mean square prediction error in the cross-validation. For both methods, the $\%{ }^{10} \mathrm{~B}$ and $\%{ }^{11} \mathrm{~B}$ in the samples were used as the Y-block components.

In order to acquire population-averaged (PA) temperature parameters, a $0.5 \% \mathrm{w} / \mathrm{w}$ amount of a potassium ferricyanide $\left(\mathrm{K}_{3}\left[\mathrm{Fe}(\mathrm{CN})_{6}\right]\right)$ dopant was added to some of the isotopically-pure boron isotopologues of $\mathrm{H}_{3} \mathrm{BO}_{3}$. The dopant was intimately mixed with the $\mathrm{H}_{3} \mathrm{BO}_{3}$ in a glass mortar and pestle before compressing into pellets. Spectra of these pellets were taken similar to those of the pure $\mathrm{H}_{3} \mathrm{BO}_{3}$ pellets, but the gate delay of the iCCD camera was set to $2 \mu$ s to measure plasma settings earlier in the lifetime of the plasma. As with the isotopic mixtures, 50 LIBS or TEPS spectra of 30 summed transients at a given position were obtained on each pellet consisting of one pure isotopologue.

The doped pellets produced spectra that contained transitions that were used for getting the plasma PA temperature (T) through the Boltzmann method [69]. In addition to the PA temperature, LIBS and TEPS electron densities were estimated from spectra collected $2 \mu s$ after plasma formation. Electron density measurements were derived from the blamer series hydrogen line $\left(\mathrm{H}_{\alpha}\right)$ near $656.3 \mathrm{~nm}$ by simultaneously monitoring the plasma of the doped samples with a second fiber-coupled iCCD camera (not shown in Figure 1) while the atomic spectra for temperature measurements were taken. The density was determined from the calculations of Gigosos and Cardeñoso [70] by interpolating from the plasma temperature and the FWHM approximated by Lorentzian fits to the normalized $\mathrm{H}_{\alpha}$ lines. Relative density measurements based on the Stark shift of the same line provided a confirmation of those obtained from the Stark width.

\section{Results and Discussion}

The BO spectra generated from LIBS and DP-LIBS for the pure boron isotopic versions of $\mathrm{H}_{3} \mathrm{BO}_{3}$ or $\mathrm{D}_{3} \mathrm{BO}_{3}$ resembled those in Figure 2. These spectra were typical of those generated by the system at the gate delay and width of the iCCD for the techniques described in the previous section. Remarkably, the spectra from the different techniques were virtually identical within the noise of the system, though the magnitude of the DP-LIBS data was about a factor of 2 higher, as detailed later. However, it was interesting to see that the trend in the relative intensities of the peaks near $261 \mathrm{~nm}$ were different for the two boron isotopes used. These spectral dissimilarities were possibly the result of slightly different rovibronic constants between the two isotopes or perhaps perturbations not accounted for in the line strength factors of the rotational transitions. The $\mathrm{D}_{3} \mathrm{BO}_{3}$ produced no noticeable spectral differences compared to $\mathrm{H}_{3} \mathrm{BO}_{3}$, so it was treated as $\mathrm{H}_{3} \mathrm{BO}_{3}$ throughout. 

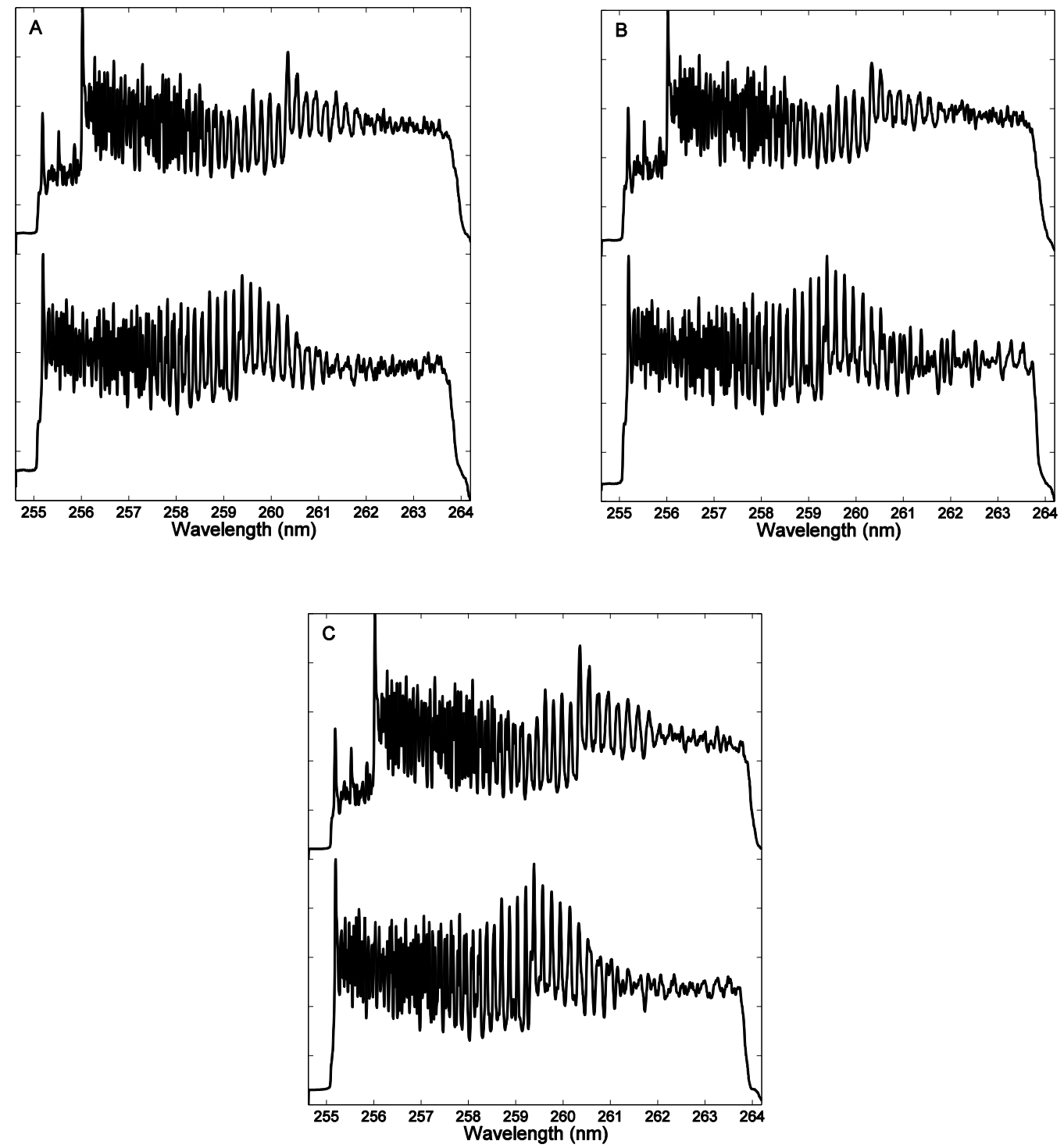

Figure 2. Exemplar spectra of LIBS (A), DP-LIBS 25 (B), and DP-LIBS 35 (C) for pure ${ }^{10} \mathrm{BO}$ (top) and ${ }^{11} \mathrm{BO}$ (bottom), generated from $\mathrm{H}_{3}{ }^{10} \mathrm{BO}_{3}$ and $\mathrm{H}_{3}{ }^{11} \mathrm{BO}_{3}$ respectively. Spectra were offset for clarity.

The LS fitting of the experimental spectra to theoretical ones were performed as the first step to data analysis. Theoretical spectra at a temperature of $4500 \mathrm{~K}$ generated by custom Matlab routines were checked for accuracy compared to those of Mélen [58]. These spectra showed similar energies and intensities as those observed in the experimental spectra of Mélen and us. Once verified as accurate, the LS fitting routines were executed on each spectrum of 30 accumulated shots to determine the $\%^{10} \mathrm{~B}$ in each series of 50 spectra on a given pellet. Plots of the predicted $\%^{10} \mathrm{~B}$ versus the measured $\%^{10} \mathrm{~B}$ were created for each technique to elucidate the accuracy of LAMIS and DP-LAMIS at different gate delays, as seen in Figure 3. Statistics from performing linear fitting of the predicted $\%^{10} \mathrm{~B}$ versus the measured $\%{ }^{10} \mathrm{~B}$, in addition to the limit 
of detection (LOD) - taken as 3 standard deviations from the mean of the blank - were also tabulated for each method in Table 2.
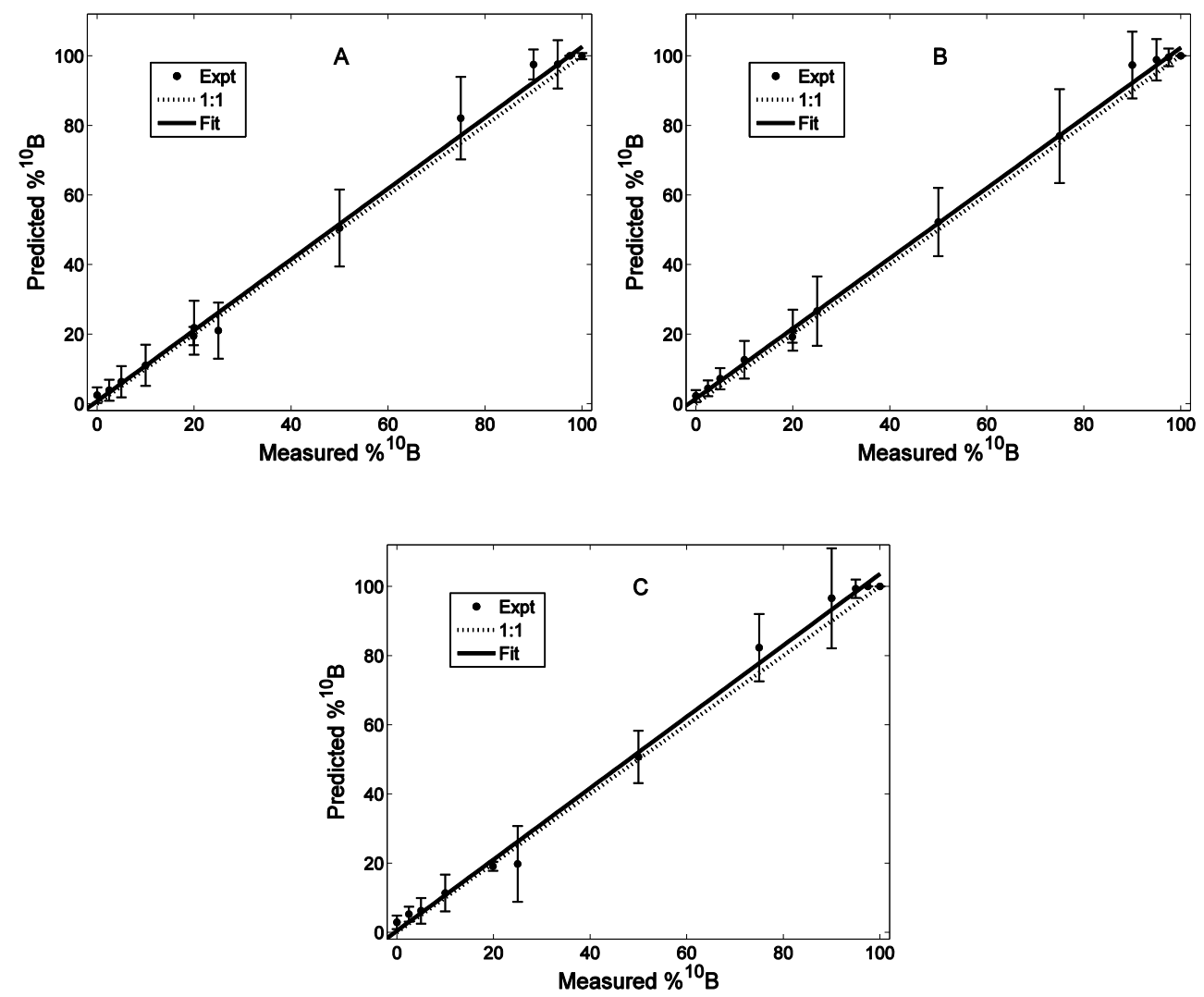

Figure 3. Plots of the predicted $\%{ }^{10} \mathrm{~B}$ versus the measured $\%{ }^{10} \mathrm{~B}$ of the $\mathrm{H}_{3} \mathrm{BO}_{3}$ samples as determined by LS-fitting of theoretical spectra to experimental ones using A) LAMIS, B) DPLAMIS with a $25 \mu \mathrm{s}$ gate delay, and C) DP-LAMIS with a $35 \mu \mathrm{s}$ gate delay. Data points are the means and error bars are the standard deviations at each concentration. The 1:1 line shows the ideal fit. 
Table 2: $\%{ }^{10} \mathrm{~B}$ Prediction Statistics

\begin{tabular}{|c|c|c|c|c|c|c|}
\hline Method & $\underline{m^{a}}$ & $\underline{b^{a}}$ & ${\underline{R^{2}}}_{\text {Cal }}{ }^{b}$ & $\underline{R}^{2} \mathrm{cv}^{\mathrm{b}}$ & ${\underline{R^{2}}}_{\text {Pred }^{b}}$ & LOD $\left(\%^{10} \mathrm{~B}\right)$ \\
\hline LAMIS (LS) & 0.982 & 1.261 & --- & --- & 0.967 & 7.7 \\
\hline DP-LAMIS $_{25}$ (LS) & 1.019 & 0.945 & --- & --- & 0.969 & 5.9 \\
\hline DP-LAMIS $_{35}$ (LS) & 1.016 & 1.250 & --- & --- & 0.974 & 6.7 \\
\hline LAMIS (PLS) & 0.964 & 0.542 & 0.978 & 0.955 & 0.960 & 6.5 \\
\hline DP-LAMIS $_{25}$ (PLS) & 0.990 & 0.175 & 0.980 & 0.977 & 0.972 & 4.9 \\
\hline DP-LAMIS $_{35}$ (PLS) & 0.995 & -0.295 & 0.990 & 0.969 & 0.975 & 6.5 \\
\hline LAMIS (CLS) & 1.003 & -1.122 & 0.972 & 0.969 & 0.971 & 10.2 \\
\hline DP-LAMIS $_{25}$ (CLS) & 1.079 & -1.275 & 0.974 & 0.973 & 0.966 & 5.1 \\
\hline DP-LAMIS $_{35}$ (CLS) & 1.026 & -0.701 & 0.976 & 0.972 & 0.966 & 12.2 \\
\hline LAMIS (LS 2) & 1.006 & -0.858 & 0.970 & --- & 0.958 & 3.0 \\
\hline DP-LAMIS $_{25}$ (LS 2) & 1.000 & -0.345 & 0.972 & --- & 0.967 & 2.4 \\
\hline DP-LAMIS $_{35}$ (LS 2) & 0.993 & -0.105 & 0.967 & --- & 0.970 & 2.3 \\
\hline
\end{tabular}

a) These parameters relate to the formula for a straight line: $y=m x+b$, where $m$ is the slope and $b$ is the offset when $\mathrm{x}$ is the measured $\%^{10} \mathrm{~B}$.

b) Cal, CV, and Pred refer to the calibration, cross-validation, and prediction DP-LIBS steps, respectively.

For comparison to the LS fits, PLS and CLS models were made for each technique to assess the quality of supervised learning methods for determining the $\%{ }^{10} \mathrm{~B}$. Each model used the first 10 spots of the collected data at a given ${ }^{10} \mathrm{~B}$ for a given gate delay and method as training, though natural abundance samples were excluded from this set because the natural abundance of boron can vary by more than one percent and the isotopologues are mixed intimately at the manufacturer, unlike the rest of the samples used in the training. Only a few spots were used in training to maximize the validation set and also to minimize the time necessary for model calibration for routine use for quantitative analysis, since it was anticipated that recalibration may be necessary once or twice daily. After calibration, plots similar to those for the LS fitting were made from the validation data, which consisted of all the spectra not included in the calibration data. These plots (shown in Figures 4 and 5), were likewise used to generate statistics about the accuracy of the fit and the LOD for each technique, as shown in Table 2. 

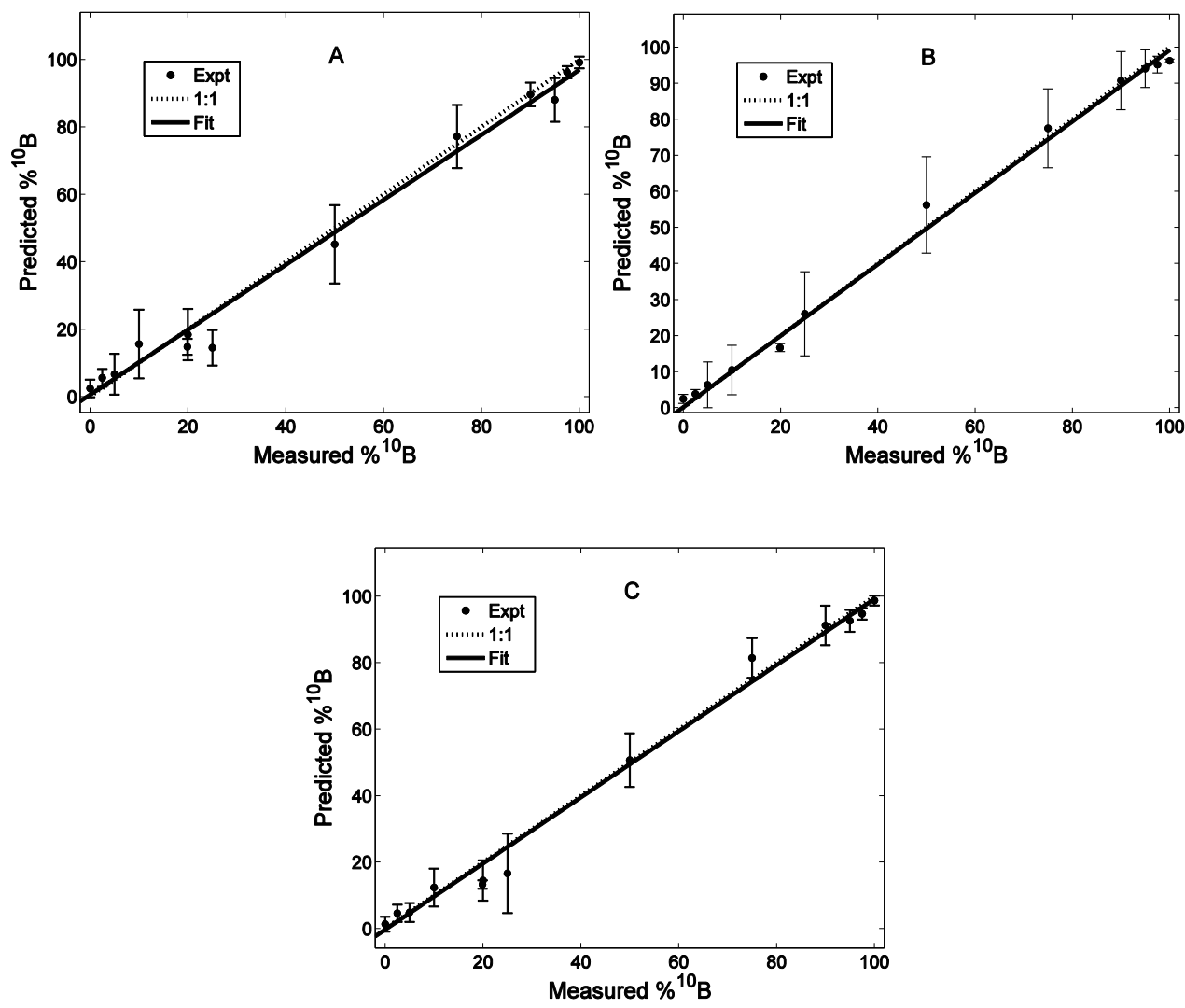

Figure 4. Plots of the predicted $\%^{10} \mathrm{~B}$ versus the measured $\%^{10} \mathrm{~B}$ of the $\mathrm{H}_{3} \mathrm{BO}_{3}$ samples as

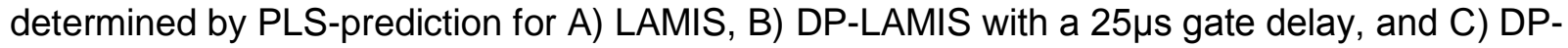
LAMIS with a $35 \mu$ gate delay. Data points are the means and error bars are the standard deviations at each concentration. The 1:1 line shows the ideal fit.
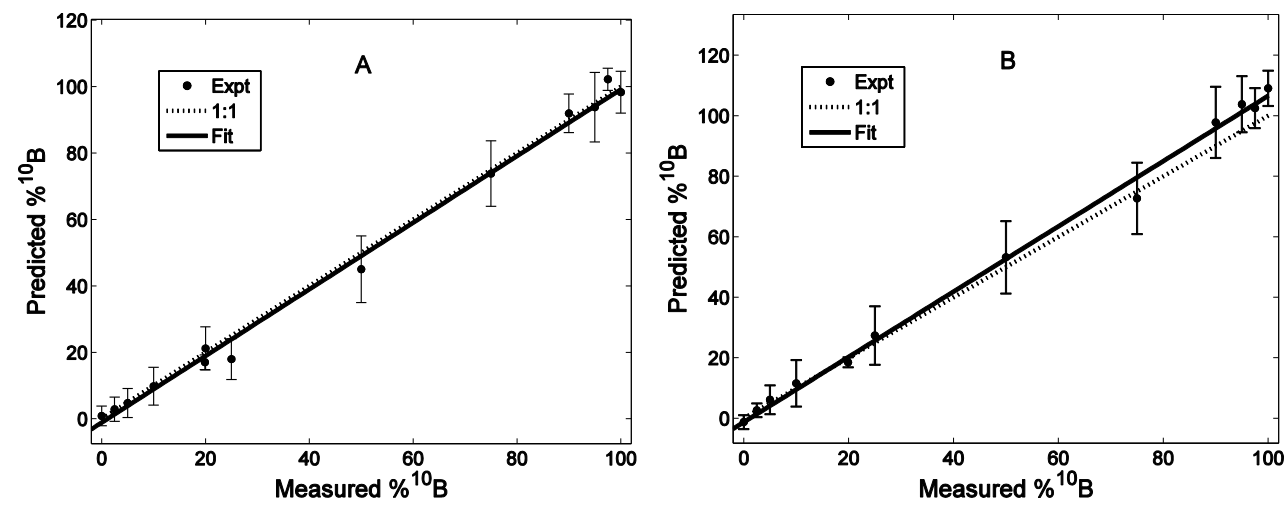


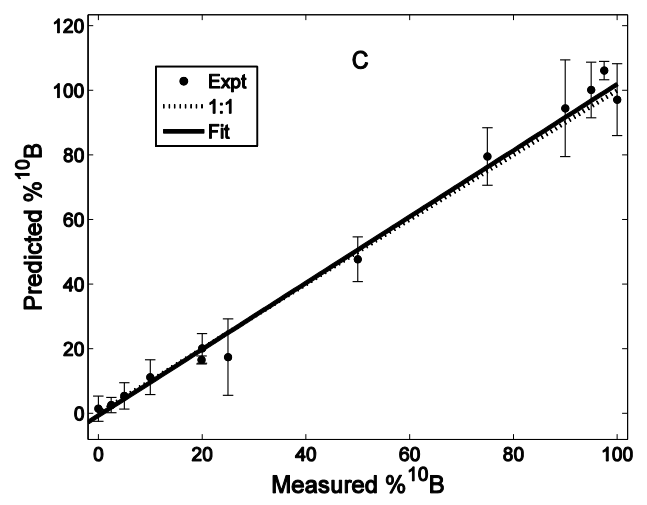

Figure 5. Plots of the predicted $\%{ }^{10} \mathrm{~B}$ versus the measured $\%{ }^{10} \mathrm{~B}$ of the $\mathrm{H}_{3} \mathrm{BO}_{3}$ samples as determined by CLS-prediction for A) LAMIS, B) DP-LAMIS with a $25 \mu$ s gate delay, and C) DPLAMIS with a $35 \mu$ s gate delay. Data points are the means and error bars are the standard deviations at each concentration. The 1:1 line shows the ideal fit.

The PLS and CLS results were expected to produce LODs less than $3 \%$, but since they did not, it was decided to try a hybrid strategy between LS and CLS. This strategy, labeled LS2 in Table 2, was performed by using the same calibration data as for CLS for the pure isotopologues, but rather than perform a decomposition during the training step, a single accumulation spectrum was formed for each isotopologue. These pure isotopologue spectra were constructed by summation of the calibration spectra for the pure isotopologues after removing outliers at every given frequency via a $Q$-test at $95 \%$ confidence [71,72]. The two outputs from this step (a single accumulation spectrum for each isotopologue), as well as a spectral offset, were then used as a basis for a least-squares fit to the same experimental calibration data as for CLS and PLS to form a linear predictor. This predictor was used to determine the concentration of the same unknown data used for CLS and PLS. Plots for the predictions of this algorithm can be found in Figure 6, and the LOD and $\mathrm{R}^{2}$ pred are in Table 2.

Figures 3 to 6 and the data in Table 2 revealed less than superb results from both LAMIS and DP-LAMIS under the conditions of the experiment. Although the $\mathrm{R}^{2}$ of the predictions was higher than 0.96 for each method except LAMIS (LS2), the LOD was about $5 \%$ or higher for all techniques except those using the LS2 algorithm. Moreover, the standard deviation at each concentration was very large for all samples but those at natural abundance (i.e., $19.9 \%{ }^{10} \mathrm{~B}$ ) and near $100 \%$ isotopically-pure B samples. These results inferred that the spectral acquisition techniques were not to blame; it was apparent from the relatively small standard deviation of the natural abundance and isotopically-pure samples that errors originated from sample inhomogeneity. Producing the calibration standards by grinding the pure isotopes together lead to standard deviations of almost $10 \%{ }^{10} \mathrm{~B}$. However, the LOD is not explained by the inhomogeneity, since it was determined mainly by the nearly pure samples of ${ }^{11} \mathrm{~B}$, having almost no ${ }^{10} \mathrm{~B}$. Instead, the high LOD likely resulted from poor fitting of theory to experiment by the algorithm in the case of LS or additionally from variations in the plasma perhaps from unoptimized instrument settings or surface inhomogeneity that affected the experimental data via noise that was unaccounted for by the PLS and CLS models. 

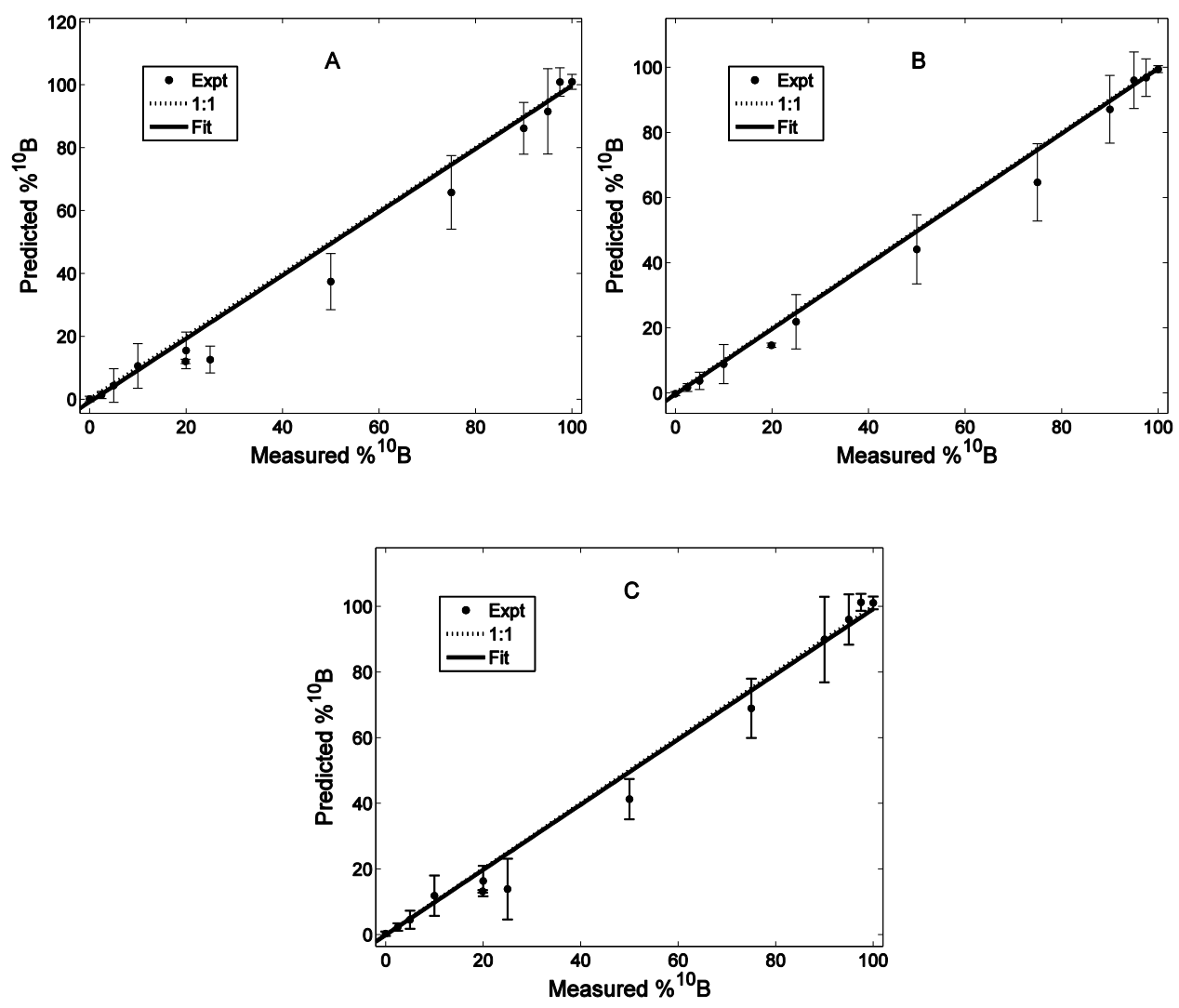

Figure 6. Plots of the predicted $\%{ }^{10} \mathrm{~B}$ versus the measured $\%{ }^{10} \mathrm{~B}$ of the $\mathrm{H}_{3} \mathrm{BO}_{3}$ samples as determined by LS2-prediction for A) LAMIS, B) DP-LAMIS with a $25 \mu$ s gate delay, and C) DPLAMIS with a $35 \mu$ gate delay. Data points are the means and error bars are the standard deviations at each concentration. The 1:1 line shows the ideal fit.

A number of objections could be raised about the findings in Table 2. For instance, it could be argued that the data appears non-linear because the range of concentrations used is too high. Though the concentrations span is large, this did not affect results in a previous study that not only used a similar concentration range, but only used even fewer sample concentrations for their models [49]. They did use many more shots in a given spot than used here, but the objective of this work was to make data acquisition fast and to work with as little material as possible. The 30 shots in a given spot used here was believed minimal to produce meaningful statistics at each location. Moreover, laser "drilling" into a sample may not be possible some cases for trace detection, such as when analyzed material is only a residue on a surface. Drilling also changes the focus depth of the laser and generates dust that may cause further inaccuracies if data is collected too quickly, since breakdown of subsequent shots may occur on the dust rather than the actual sample. Some of this dust breakdown may have affected the results in Table 2, though it was not evident during data collection, as variation between the first 10 shots and the last 10 shots appeared minimal. Finally, these results were not specific to $\mathrm{H}_{3} \mathrm{BO}_{3}$; a small amount of similar data collected on boron oxide $\left(\mathrm{B}_{2} \mathrm{O}_{3}\right)$ with similar instrument settings summing 50 shots at each sample location produced nearly identical results to those in Table 2. 
Because DP-LAMIS did not radically improve the accuracy of the determination of isotopes, factors affecting the plasmas were considered. In particular, the differences in PA temperature between LAMIS and DP-LAMIS shortly after the plasma was first formed were determined by examining the spectra generated from the doped samples. The $\mathrm{K}_{3}\left[\mathrm{Fe}(\mathrm{CN})_{6}\right]$ used in the doped samples here were capable of providing multiple intense transitions that were easy to observe within the wavelength band pass of the spectrometer. Neutral lines of metals were then used with the Boltzmann-type plots to determine the PA temperature.

Matlab LS routines for fitting the Boltzmann equation were performed to get the PA temperature from Fe (II) transitions. Accept for the intensities of the Fe (II) lines, the parameters for the equation were obtained from the NIST atomic spectroscopy database [73]. The features that were used - shown in Table 3 - were selected based on spectral intensities and on the separation between the upper-state energies being sufficient to ensure adequate leveraging for LS fitting.

Table 3: Fe (II) transitions used for temperature calculations

\begin{tabular}{cccc}
\hline $\boldsymbol{\lambda}(\mathbf{n m})$ & $\boldsymbol{A}_{\text {ik }}\left(\mathbf{1 0}^{\mathbf{8}} \mathbf{s}^{-1}\right)$ & $E_{\mathrm{k}}(\mathbf{e V})$ & $\boldsymbol{g}_{\mathrm{k}}$ \\
\hline 271.184 & 0.440 & 7.723 & 14 \\
271.441 & 0.570 & 5.552 & 8 \\
271.622 & 1.150 & 7.988 & 6 \\
272.754 & 0.938 & 5.585 & 4 \\
273.697 & 1.220 & 5.605 & 2 \\
274.320 & 1.970 & 5.615 & 4 \\
\hline
\end{tabular}




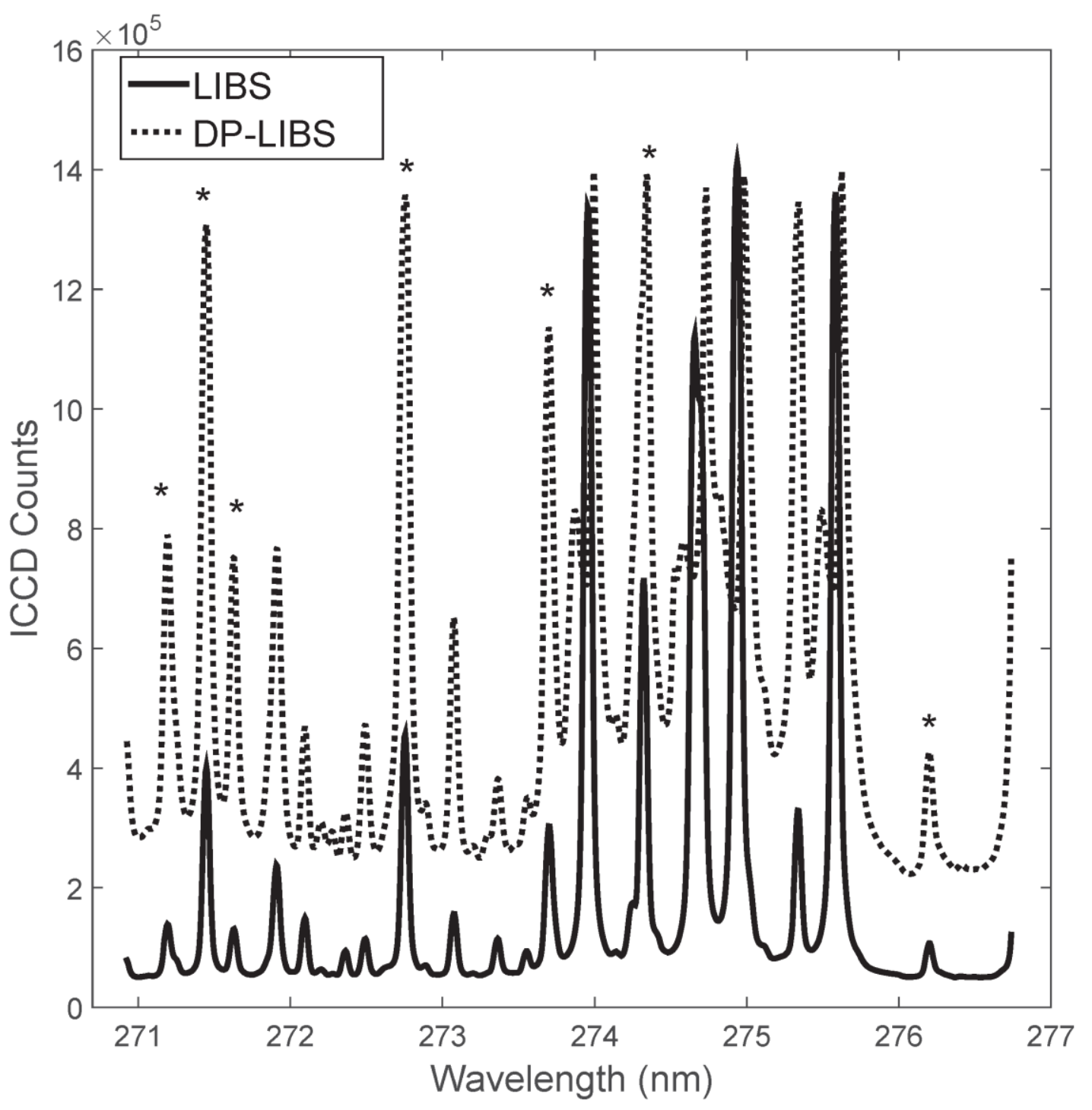

Figure 7. The iron spectra for LIBS and DP-LIBS $2 \mu$ after the $\mathrm{Nd}^{3+}:$ YAG laser formed a plasma on $\mathrm{Fe}$-doped $\mathrm{H}_{3} \mathrm{BO}_{3}$. Features marked with stars were used to calculate a PA temperature via Equation 1.

The PA temperature calculations indicated that the DP-LIBS plasmas were hotter by a factor of 1.25 to 1.5, as seen in Table 4. Even so, it was believed that these measurements did not represent a uniform temperature of the plasma. Limited data from Fe (I) lines in Figure 7 (not labeled), which were somewhat weak, showed a plasma temperature about half the Fe (II) determined temperature. Errors in the fits were likely due to inaccuracies in determining the transition intensities, since a sloping baseline, taken as the minimum over a $\pm 1 \mathrm{~nm}$ window centered on each transition, was subtracted from each measured intensity. Because the sloping baseline was worse for DP-LIBS, it is possible that the PA temperature may be even higher than that reported here. In addition, these temperatures should be viewed with caution as the small 
amounts of material added to the samples may have caused significant matrix effects, though the data here does allow DP-LIBS and LIBS to be compared relative to each other. In addition, local thermodynamic equilibrium (LTE) has been assumed and not proven. Demonstrating the LTE condition was difficult so the temperature measurements were circumspect. Still, the spectra clearly show a number of ions present for DP-LIBS that are missing with LIBS, seemingly indicating that the temperature is higher for DP-LIBS.

Table 4: Mean PA Temperature and Electron Density Measurements on $\mathrm{H}_{3} \mathrm{BO}_{3}$ Isotopologes

\begin{tabular}{cccc}
\hline Material & $\begin{array}{c}\text { Excitation } \\
\text { Modality }\end{array}$ & Temperature $\left(\mathbf{1 0} \mathbf{K}^{\mathbf{a}}\right.$ & Electron Density $\left(\mathbf{1 0}^{\mathbf{1 7}} \mathbf{c m}^{-3} \mathbf{b}^{\mathbf{b}}\right.$ \\
\hline $\mathrm{H}_{3}{ }^{10} \mathrm{BO}_{3}$ & LIBS & $2.14(0.31)$ & $1.64(0.48)$ \\
$\mathrm{H}_{3}{ }^{10} \mathrm{BO}_{3}$ & DP-LIBS & $3.02(0.34)$ & $4.02(1.37)$ \\
$\mathrm{H}_{3}{ }^{11} \mathrm{BO}_{3}$ & LIBS & $2.20(0.39)$ & $2.25(0.74)$ \\
$\mathrm{H}_{3}{ }^{11} \mathrm{BO}_{3}$ & DP-LIBS & $2.75(0.29)$ & $3.27(0.56)$ \\
\hline
\end{tabular}

a) Numbers in parentheses are standard deviations

b) Numbers in parentheses are standard deviations

Attempts were also made at obtaining the average electron densities (Table 4) from Stark width and shift measurements of the hydrogen $\mathrm{H}_{\alpha}$ line at $656.27 \mathrm{~nm}$ (Figure 8) using the same times as the PA temperatures for all 50 spectra on a given pellet. Tables of electron densities at different temperatures and Stark widths from [70], as well as different ion contributions (determined by a parameter $\mu$ ) provided more convenient and perhaps more accurate densities than those from Griem's method [74]. Unfortunately, influence from the ions was uncertain, so the value of $\mu=1.0$ was used for table interpolation in [70]; this value corresponded to a hydrogen emitter in the presence of heavy ions. 


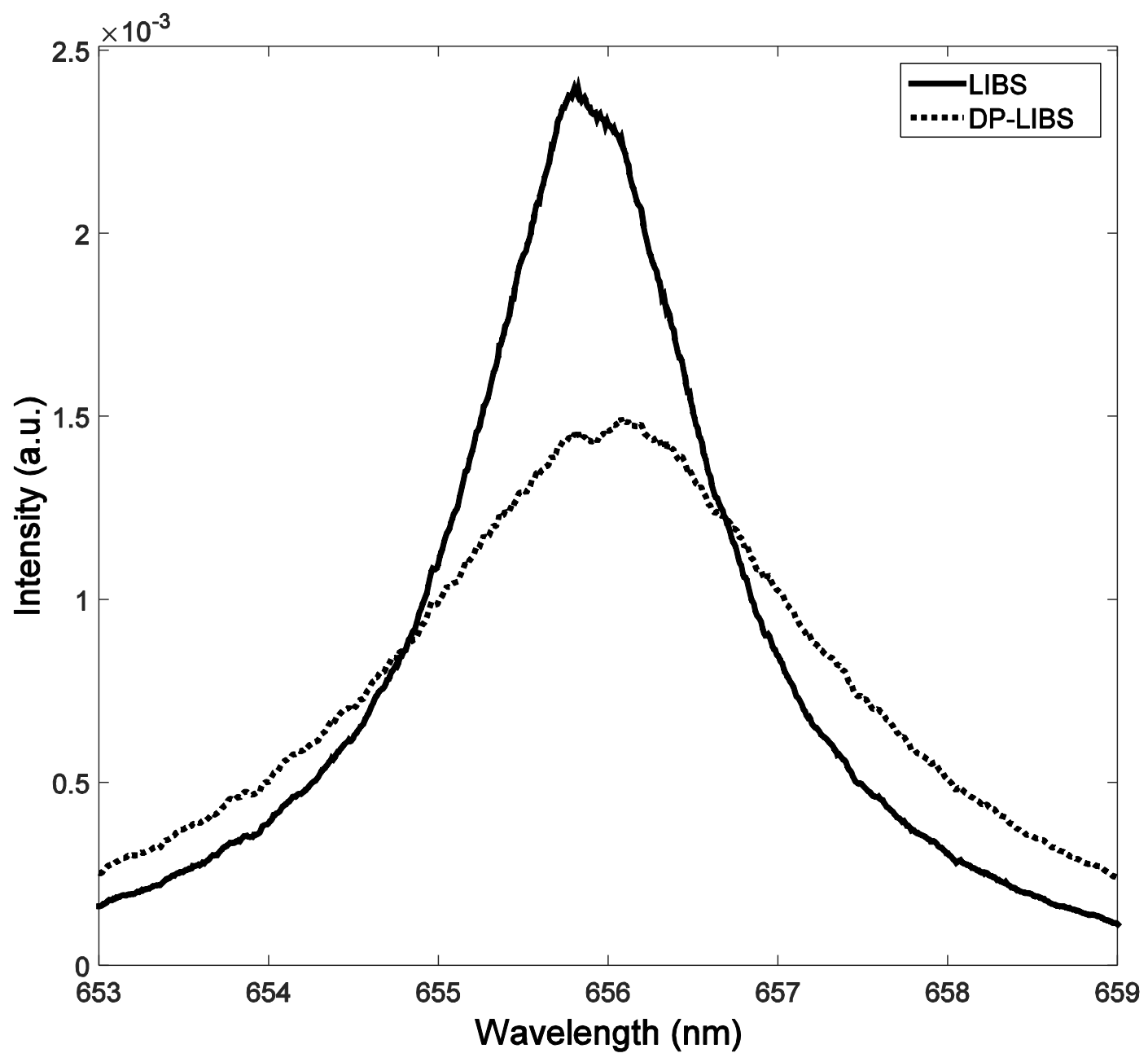

Figure 8. The $\mathrm{H}_{\alpha}$ line at $656.27 \mathrm{~nm}$ for LIBS and DP-LIBS $2 \mu$ after the $\mathrm{Nd}^{3+}:$ YAG laser formed a plasma on Fe-doped $\mathrm{H}_{3} \mathrm{BO}_{3}$.

In general, the DP-LAMIS plasmas were a factor of 1.5 to 2.5 more electrons dense than the LAMIS plasmas from Stark widths, which concurred with the DP-LAMIS/LAMIS ratios obtained from the Stark shifts. However, these widths were on par with those of Parigger at a few ns in their plasmas of pure hydrogen created using even more energy per pulse [75]. It was expected that hydrogen gas in Parigger's work would actually provide much higher electron densities, considering no energy was needed to ablate a hydrogen gas sample. Consequently, the actual number densities of electrons were viewed as circumspect, and only the DP-LAMIS/LAMIS ratio was considered somewhat valid.

Foregoing absolute electron densities, the increased relative density of DP-LAMIS plasmas suggested they could have contained more BO, since the larger number of electron collisions with atoms in the air would create more chemical reactions in the plasma. Indeed, DP-LAMIS BO spectra were more intense than those from LAMIS by a factor of 2 for DP-LAMIS 25 and by about a factor of 1.5 for DP-LAMIS 35 . Because the calculated molecular temperatures for the 
plasmas for both LAMIS and DP-LAMIS were approximately the same at the time the BO spectra were taken, at least according to the LS-fits, it was believed that DP-LIBS formed more $\mathrm{BO}$ that mediated the cooling of the plasma relative to that of LIBS at the gate delays at which the BO spectra were collected, though this is difficult to say with certainty.

It should be noted that all results obtained here were a prelude to standoff detection of nuclear materials. It was certainly obvious that orthogonal methods could not be used for standoff techniques, but the objective for this work was to determine the effect of the mid-IR laser on the plasma under equipment settings that might be applied to standoff detection at a later time. The orthogonal enhancement did show some marginal promise for improved detection. However, the linearity of the model was problematic, and this aspect of detection was deemed likely to get worse at standoff ranges where precise beam alignment may be difficult to achieve. Using these results alone, it was uncertain if DP-LIBS with a mid-IR laser for the enhancing pulse might be useful for standoff detection of isotopologues.

Finally, it seems apparent that further research into DP-LAMIS is necessary to determine the underlying causes for the lack of improvement in LOD seen here. The difference between the two techniques appears to not be related to a lack of plasma emission for DP-LAMIS. It is possible that the TEA laser may have hot spots that are not reproducible from shot to shot so that while a hotter or larger plasma is formed, it is no better from an analytical standpoint than a LAMIS plasma. In addition, the models used data collected over days. Instrumental and environmental conditions can change over time so that these variations may dominate over any benefit gained from the double-pulse technique. Still, the fact that the intensity is higher with DPLAMIS makes it useful if it extends to a collinear application.

\section{Conclusion}

The LAMIS method for determining the isotopic composition of a sample has been tested with a LIBS enhancement technique called DP-LIBS. DP-LIBS was applied to LAMIS via the use of a $\mathrm{CO}_{2}$ TEA laser pulse fired shortly after the LAMIS plasma was created. The DP-LIBS enhancement to LAMIS was termed DP-LIBS-enhanced LAMIS or DP-LAMIS.

To determine the possible improvements of DP-LAMIS over LAMIS, boron-isotopologue mixtures of $\mathrm{H}_{3} \mathrm{BO}_{3}$ were analyzed with both methods for the relative ${ }^{10}{ }^{10} \mathrm{~B}$ abundance using samples containing from $0 \%$ to essentially $100 \%{ }^{10} \mathrm{~B}$. Fifty spectra of 30 accumulated transients were analyzed at different concentrations using a calibration-free, LS model; CLS; PLS; and a new least-squares algorithm called LS2. Results of these analyses were tabulated and compared to determine if DP-LAMIS, performed using gate delays of 25 and $35 \mu$ s after the plasma formed, had any advantage over LAMIS using a gate delay of $25 \mu \mathrm{s}$.

Both DP-LAMIS and LAMIS showed similar trends with concentration in all models used. In general, the mean predicted concentrations of $\%^{10} \mathrm{~B}$ were within $5 \%$ of the measured concentrations for all models except CLS, and in some cases, were much lower. However, the standard deviation at each concentration was larger than expected for even the single isotopologue and natural abundance samples where no mixing of isotopologues was performed, except for the LS2 method. Consequently, the limit of detection for all models was far higher 
than was deemed acceptable except for the DP-LAMIS approaches with LS2 fits where the limit of detection was closer to 2.3 to $2.4 \%$ for the $\%^{10} \mathrm{~B}$ isotopologue, which is slightly lower than previous LAMIS work.[1]

Finally, plasma temperature and electron density measurements were made using samples doped with materials containing metal atoms in order to determine the differences in the plasma conditions for LAMIS and DP-LAMIS early in the plasma history. These efforts showed that the plasma is about a factor of 2 hotter for DP-LAMIS initially, as well as being 1.3 to 2 times as dense compared to LAMIS. Consequently, it appeared that the DP-LAMIS plasma cooled faster though it generated more BO signal for both MIRELAMIS ${ }_{25}$ DP-LAMIS and MIRELAMIS ${ }_{35}$ DPLAMIS, indicating that the conditions early in the plasma history can impact the sensitivity of LAMIS and DP-LAMIS analyses.

\section{Acknowledgements}

The authors wish to thank the Defense Threat Reduction Administration (DTRA) of the U. S. Department of Defense for their support for this work under contract HDTRA1-12-1-0036.

\section{References}

[1] R.E. Russo, A.A. Bol'shakov, X. Mao, C.P. McKay, D.L. Perry, O. Sorkhabi, Laser Ablation Molecular Isotopic Spectrometry, Spectrochim. Acta Part B At. Spectrosc. 66 (2011) 99-104. doi:10.1016/j.sab.2011.01.007.

[2] X. Mao, A.A. Bol'shakov, D.L. Perry, O. Sorkhabi, R.E. Russo, Laser Ablation Molecular Isotopic Spectrometry: Parameter influence on boron isotope measurements, Spectrochim. Acta Part B At. Spectrosc. 66 (2011) 604-609. doi:10.1016/j.sab.2011.06.007.

[3] X. Mao, A.A. Bol'shakov, I. Choi, C.P. McKay, D.L. Perry, O. Sorkhabi, and Russo, Richard E., Laser Ablation Molecular Isotopic Spectrometry: Strontium and its isotopes, Spectrochim. Acta Part B At. Spectrosc. 66 (2011) 767-775. doi:10.1016/j.sab.2011.12.002.

[4] J.E. Boggs, Nuclear Vibrations and Force Constants, in: Z.B. Maksić (Ed.), Theor. Model. Chem. Bond. - Mol. Spectrosc. Electron. Struct. Intramol. Interact. Part 3, Springer Berlin Heidelberg, Berlin, Heidelberg, 1991: pp. 1-24. doi:10.1007/978-3-642-58179-3.

[5] H.W. Kroto, Molecular Rotation Spectra, 2nd ed., Dover, Mineola, N. Y., 2003.

[6] R.S. Mulliken, The Isotope Effect in Band Spectra, II: The Spectrum of Boron Monoxide, Phys. Rev. 25 (1925) 259-294. doi:10.1103/PhysRev.25.259.

[7] G. Herzberg, Molecular Spectra and Molecular Structure. I. Spectra of Diatomic Molecules, 2nd ed., Krieger, 1966. doi:10.1119/1.1932852.

[8] J. Uebbing, J. Brust, W. Sdorra, F. Leis, K. Niemax, Reheating of a \{Laser-Produced\} Plasma by a Second Pulsed Laser, Appl. Spectrosc. 45 (1991) 1419-1423.

[9] R. Nyga, W. Neu, Double-Pulse Technique for Optical Emission Spectroscopy of Ablation Plasmas of Samples in Liquids, Opt. Lett. 18 (1993) 747-749.

[10] V.I.I. Babushok, F.C. DeLucia, J.L.L. Gottfried, C. a. A. Munson, a. W.W. Miziolek, Double pulse laser ablation and plasma: Laser induced breakdown spectroscopy signal enhancement, Spectrochim. Acta - Part B At. Spectrosc. 61 (2006) 999-1014. 
doi:10.1016/j.sab.2006.09.003.

[11] R.C. Chinni, D.A. Cremers, L.J. Radziemski, M. Bostian, C. Navarro-Northrup, Detection of Uranium Using Laser-Induced Breakdown Spectroscopy, Appl. Spectrosc. 63 (2009) 1238-1250. http://as.osa.org/abstract.cfm?URI=as-63-11-1238 (accessed June 24, 2013).

[12] R. Ahmed, M.A. Baig, A comparative study of single and double pulse laser induced breakdown spectroscopy, J. Appl. Phys. 106 (2009) 033307. doi:10.1063/1.3190516.

[13] M.E. Asgill, M.S. Brown, K. Frische, W.M. Roquemore, D.W. Hahn, Double-pulse and single-pulse laser-induced breakdown spectroscopy for distinguishing between gaseous and particulate phase analytes, Appl. Opt. (2010). doi:10.1364/AO.49.00C110.

[14] J.L. Gottfried, F.C. De Lucia, Laser-Induced Breakdown Spectroscopy: Capabilities and Applications, 2010.

[15] K. Dzierżęga, a Mendys, S. Pellerin, E. Thouin, G. Travaille, B. Bousquet, Canioni, L., and Pokrzywka, B., Thomson scattering from laser induced plasma in air, J. Phys. Conf. Ser. 227 (2010) 012029. doi:10.1088/1742-6596/227/1/012029.

[16] L. St-Onge, V. Detalle, M. Sabsabi, Enhanced Laser Induced Breakdown Spectroscopy Using the Combination of Fourth-Harmonic and Fundamental Nd:YAG Laser Pulses, Spectrochim. Acta Part B. 57 (2002) 121-135.

[17] V.N. Rai, F.Y. Yueh, J.P. Singh, Time-dependent single and double pulse laser-induced breakdown spectroscopy of chromium in liquid, Appl. Opt. 47 (2008) G21. doi:10.1364/AO.47.000G21.

[18] D.N. Stratis, K.L. Eland, S.M. Angel, Dual-pulse LIBS: why are two lasers better than one?, in: T. Vo-Dinh, R.L. Spellicy (Eds.), Proc. SPIE 3853, Environ. Monit. Remediat. Technol. II, 1999: pp. 385-392. doi:10.1117/12.372877.

[19] G. Galbács, V. Budavári, Z. Geretovszky, Multi-pulse laser-induced plasma spectroscopy using a single laser source and a compact spectrometer, J. Anal. At. Spectrom. 20 (2005) 974. doi:10.1039/b504373e.

[20] Z. Hou, Z. Wang, J. Liu, W. Ni, Z. Li, Signal quality improvement using cylindrical confinement for laser induced breakdown spectroscopy, 21 (2013) 15974-15979. doi:10.1364/OE.21.015974.

[21] D.N. Stratis, K.L. Eland, S.M. Angel, Dual-Pulse LIBS Using a Pre-ablation Spark for Enhanced Ablation and Emission, Appl. Spectrosc. 54 (2000) 1270-1274.

[22] D.N. Stratis, K.L. Eland, S.M. Angel, Enhancement of Aluminum, Titanium, and Iron in Glass Using Pre-ablation Spark Dual-Pulse LIBS, Appl. Spectrosc. 54 (2000) 1719-1726. doi:10.1366/0003702001948871.

[23] C. Guatier, P. Fichet, D. Menut, J. Dubessy, Applications of the Double Pulse Laser Induced Breakdown Spectroscopy $\{($ LIBS $)\}$ in the Colinear Beam Geometry to the Elemental Analysis of Different Materials, Spectrochim. Acta Part B. 61 (2006) 210-219.

[24] C. Guatier, P. Fichet, D. Menut, J.L. Lacour, D. L'Hermite, J. Dubessy, Study of the Double Pulse Setup with an Orthogonal Beam Geometry for Laser Induced Breakdown Spectroscopy, Spectrochim. Acta Part B. 59 (2004) 975-986.

[25] C. Guatier, P. Fichet, D. Menut, J.L. Lacour, D. L'Hermite, J. Dubessy, Quantification of the Intensity Enhancements for the Double Pulse Laser Induced Breakdown Spectroscopy (LIBS) in the Orthogonal Beam Geometry, Spectrochim. Acta Part B. 60 (2005) 792-804. 
[26] F.C. De Lucia, J.L. Gottfried, C. a. Munson, A.W. Miziolek, Double pulse laser-induced breakdown spectroscopy of explosives: Initial study towards improved discrimination, Spectrochim. Acta Part B At. Spectrosc. 62 (2007) 1399-1404. doi:10.1016/j.sab.2007.10.036.

[27] K. Amal, S.H.H. Elnaby, V. Palleschi, a. Salvetti, M. a. A. Harith, Comparison between single- and double-pulse LIBS at different air pressures on silicon target, Appl. Phys. B. 83 (2006) 651-657. doi:10.1007/s00340-006-2259-1.

[28] A.J. Effenberger, J.R. Scott, Effect of atmosphere on collinear double-pulse laser-induced breakdown spectroscopy., Anal. Bioanal. Chem. 400 (2011) 3217-27. doi:10.1007/s00216-011-5034-z.

[29] P.K. Diwakar, S.S. Harilal, J.R. Freeman, a. Hassanein, Role of laser pre-pulse wavelength and inter-pulse delay on signal enhancement in collinear double-pulse laserinduced breakdown spectroscopy, Spectrochim. Acta Part B At. Spectrosc. 87 (2013) 6573. doi:10.1016/j.sab.2013.05.015.

[30] D. Killinger, S.D. Allen, R.D. Waterbury, C. Stefano, E.L. Dottery, Enhancement of Nd:YAG LIBS Emission of a Remote Target Using a Simultaneous CO2 Laser Pulse, Opt. Express. 15 (2007) 12905-12915.

[31] A. Pal, R.D. Waterbury, E.L. Dottery, D.K. Killinger, Enhanced temperature and emission from a standoff $266 \mathrm{~nm}$ laser initiated LIBS plasma using a simultaneous $10.6 \mu \mathrm{m} \mathrm{CO} 2$ laser pulse, Opt. Express. 17 (2009) 8856. doi:10.1364/OE.17.008856.

[32] R.W. Coons, S.S. Harilal, S.M. Hassan, A. Hassanein, The importance of longer wavelength reheating in dual-pulse laser-induced breakdown spectroscopy, Appl. Phys. B. 107 (2012) 873-880. doi:10.1007/s00340-012-4997-6.

[33] A. Ford, R. Waterbury, J. Rose, K. Pohl, M. Eisterhold, T. Thorn, Lee, K., and Dottery, E., Extension of a standoff explosive detection system to CBRN threats, in: A.W. Fountain III, P.J. Gardner (Eds.), Proc. SPIE, 2010: p. 76650Y-76650Y-10. doi:10.1117/12.849815.

[34] M. Weidman, S. Palanco, M. Baudelet, M.C. Richardson, Thermodynamic and spectroscopic properties of Nd:YAG-CO2 Double-Pulse Laser-Induced Iron Plasmas, Spectrochim. Acta Part B At. Spectrosc. 64 (2009) 961-967. doi:10.1016/j.sab.2009.07.023.

[35] J. Register, J. Scaffidi, S.M. Angel, Direct measurements of sample heating by a laserinduced air plasma in pre-ablation spark dual-pulse laser-induced breakdown spectroscopy (LIBS)., Appl. Spectrosc. 66 (2012) 869-74. doi:10.1366/11-06531.

[36] S.S. Harilal, P.K. Diwakar, a. Hassanein, Electron-ion relaxation time dependent signal enhancement in ultrafast double-pulse laser-induced breakdown spectroscopy, Appl. Phys. Lett. 103 (2013) 041102. doi:10.1063/1.4816348.

[37] V. Piscitelli S, M. a. Martínez L., a. J. Fernández C., J.J. González, X.L. Mao, R.E. Russo, Double pulse laser induced breakdown spectroscopy: Experimental study of lead emission intensity dependence on the wavelengths and sample matrix, Spectrochim. Acta Part B At. Spectrosc. 64 (2009) 147-154. doi:10.1016/j.sab.2008.11.008.

[38] N.W. Jalufka, Laser Production and Heating of Plasma for MHD Application, in: NASA TP-2798, NASA, Hampton, VA, 1988: pp. 1-8.

[39] M. Weidman, M. Baudelet, S. Palanco, M. Sigman, P.J. Dagdigian, M. Richardson, Nd:YAG-CO2 double-pulse laser induced breakdown spectroscopy of organic films, Opt. Express. 18 (2010) 259-66. http://www.ncbi.nlm.nih.gov/pubmed/20173846. 
[40] F.C. De Lucia, J.L. Gottfried, C.A. Munson, A.W. Miziolek, Double pulse laser-induced breakdown spectroscopy of explosives: Initial study towards improved discrimination, Spectrochim. Acta Part B. 67 (2007) 1399-1404.

[41] M. Gaft, L. Nagli, N. Eliezer, Y. Groisman, O. Forni, Elemental analysis of halogens using molecular emission by laser-induced breakdown spectroscopy in air, Spectrochim. Acta Part B At. Spectrosc. 98 (2014) 39-47. doi:10.1016/j.sab.2014.05.011.

[42] X. Liu, S. Sun, X. Wang, Z. Liu, Q. Liu, P. Ding, Guo, Z., and Hu, B., Effect of laser pulse energy on orthogonal double femtosecond pulse laser-induced breakdown spectroscopy, Opt. Express. 21 (2013) A704. doi:10.1364/OE.21.00A704.

[43] G. Velarde, Y. Ronen, J.M. Martinez-Val, Nuclear Fusion by Inertial Confinement, CRC Press, Boca Raton, FL, 1993.

[44] S.S. Harilal, T. Sizyuk, a. Hassanein, D. Campos, P. Hough, V. Sizyuk, The effect of excitation wavelength on dynamics of laser-produced tin plasma, J. Appl. Phys. 109 (2011) 063306. doi:10.1063/1.3562143.

[45] A.M. Rubenchik, On the Initiation of High Explosives by Laser Radiation, Propellants, Explos. Pyrotech. 32 (2007) 296-300. doi:10.1002/prep.200700031.

[46] N.K. Bourne, On the laser ignition and initiation of explosives, Proc. R. Soc. A Math. Phys. Eng. Sci. 457 (2001) 1401-1426. doi:10.1098/rspa.2000.0721.

[47] L. Strakovskiy, A. Cohen, R. Fifer, R. Beyer, B. Forch, Laser Ignition of Propellants and Explosives, Aberdeen Proving Ground, MD, 1998.

[48] V. Contreras, M.A. Meneses-Nava, O. Barbosa-García, J.L. Maldonado, G. Ramos-Ortiz, Double-pulse and calibration-free laser-induced breakdown spectroscopy at low-ablative energies., Opt. Lett. 37 (2012) 4591-3. http://www.ncbi.nlm.nih.gov/pubmed/23164848 (accessed October 8, 2013).

[49] A. Sarkar, X. Mao, R.E. Russo, Advancing the analytical capabilities of laser ablation molecular isotopic spectrometry for boron isotopic analysis, Spectrochim. Acta Part B At. Spectrosc. 92 (2014) 42-50. doi:10.1016/j.sab.2013.12.001.

[50] J. Dunham, The Energy Levels of a Rotating Vibrator, Phys. Rev. 41 (1932) 721-731. doi:10.1103/PhysRev.41.721.

[51] J.T. Vanderslice, E.A. Mason, W.G. Maisch, E.R. Lippincott, Ground state of hydrogen by the Rydberg-Kelein-Rees method, J. Mol. Spectrosc. 3 (1959) 17-29. doi:10.1016/00222852(59)90003-7.

[52] N.L. Singh, D.C. Jain, The Rydberg-Klein-Rees Method of Constructing the True Potential Energy Curves of Diatomic Molecules, Proc. Phys. Soc. 79 (1962) 274-278. doi:10.1088/0370-1328/79/2/304.

[53] S. Chandra, Relation between spectroscopic constants with limited Dunham coefficients, Pramana. 65 (2005) 1133-1137. doi:10.1007/BF02705289.

[54] N. Inostroza, J.R. Letelier, M.L. Senent, On the numerical determination of Dunham's coefficients: An application to X1 $1+\mathrm{HCl}$ isotopomers, J. Mol. Struct. THEOCHEM. 947 (2010) 40-44. doi:10.1016/j.theochem.2010.01.037.

[55] M.L. da Silva, Guidelines for the Calculation of Bound Molecular Spectra, 1 (2005) 17. http://arxiv.org/abs/physics/0509132 (accessed August 27, 2014).

[56] T.A. Blake, J.F. Kelly, N.B. Gallagher, P.L. Gassman, T.J. Johnson, Passive standoff detection of RDX residues on metal surfaces via infrared hyperspectral imaging, Anal. 
Bioanal. Chem. (2009) 337-348. doi:10.1007/s00216-009-2907-5.

[57] C. Pasquini, J. Cortez, L.M.C. Silva, F.B. Gonzaga, Laser Induced Breakdown Spectroscopy, J. Braz. Chem. Soc. 18 (2007) 463-512.

[58] F. Mélen, I. Dubois, H. Bredohl, The A-X and B-X transitions of BO, J. Phys. B At. Mol. Opt. Phys. 2423 (1985) 2423-2432.

[59] V.N. Ochkin, Spectroscopy of Low Temperature Plasmas, Wiley-VCH Verlag GmbH \& Co. KGaA, Weinheim, Germany, 2009. doi:10.1002/9783527627509.

[60] H.S. Liszt, W.M.H. Smith, RKR Franck-Condon Factors for Blue and Ultraviolet Transitions of Some Metal Oxides, J. Quant. Spectrosc. Radiat. Transf. 11 (1971) 10431062.

[61] D.W. Berreman, Unfolding spectrometer slit broadening effects from broad spectra., Appl. Opt. 7 (1968) 1447-53. http://www.ncbi.nlm.nih.gov/pubmed/20068823 (accessed October 2, 2013).

[62] D. Bulajic, M. Corsi, G. Cristoforetti, S. Legnaioli, V. Palleschi, A. Salvetti, and Tognoni, E., Spectrochim. Acta Part B. 57 (2002) 339-353.

[63] V.N. Rai, S.N. Thakur, Physics of Plasma in Laser-Induced Breakdown Spectroscopy, in: J.P. Singh, S.N. Thakur (Eds.), Laser-Induced Break. Spectrosc., Elsevier B.V., Oxford, 2007: pp. 84-111.

[64] R.M. Herman, Unified Impact Theory for Velocity-Changing Effects and Speed Dependencies in Neutral Species Lineshapes, Int. J. Spectrosc. 2010 (2010) 1-9. doi:10.1155/2010/306392.

[65] C. Wang, Cavity Ringdown Spectroscopy of Plasma Species, in: P.K. Chu, X. Lu (Eds.), Low Temp. Plasma Technol. Methods Appl., CRC Press, Boca Raton, FL, 2014: pp. 207260.

[66] The MathWorks Inc., MATLAB and Statistics Toolbox Release 2012b, (2012).

[67] S. Brown, A. Ford, C.C. Akpovo, J. Martinez, L. Johnson, Matrix effects in laser ablation molecular isotopic spectrometry, Spectrochim. Acta Part B At. Spectrosc. 101 (2014) 204-212. doi:10.1016/j.sab.2014.09.003.

[68] Eigenvector Research Inc., PLS Toolbox 7.5, (2013).

[69] J.A. Aguilera, C. Aragon, Apparent excitation temperature in laser-induced plasmas, J. Phys. Conf. Ser. 59 (2007) 210-217. http://www.iop.org/EJ/abstract/1742-6596/59/1/046.

[70] M. a Gigosos, V. Cardeñoso, New plasma diagnosis tables of hydrogen Stark broadening including ion dynamics, J. Phys. B At. Mol. Opt. Phys. 29 (1996) 4795-4838. doi:10.1088/0953-4075/29/20/029.

[71] R.B. Dean, W.J. Dixon, Simplified Statistics for Small Numbers of Observations, Anal. Chem. 23 (1951) 636-638. doi:10.1021/ac60052a025.

[72] D.B. Rorabacher, Statistical Treatment for Rejection of Deviant Values : Critical Values of Dixon ' s " $Q$ " Parameter and Related Subrange Ratios at the $95 \%$ Confidence Level, Test. (1991) 139-146.

[73] A. Kramida, Y. Ralchenko, J. Reader, NIST ASD Team, NIST Atomic Spectra Database (ver. 5.1), [Online], Natl. Inst. Stand. Technol. MD. (2013) http://physics.nist.gov/asd. http://physics.nist.gov/asd.

[74] H.R. Griem, Plasma Spectroscopy, McGraw-Hill, New York, 1964. 
[75] C.G. Parigger, Atomic and molecular emissions in laser-induced breakdown spectroscopy, Spectrochim. Acta Part B At. Spectrosc. 79-80 (2013) 4-16. doi:10.1016/j.sab.2012.11.012. 